\title{
Chapter 10 \\ What can a mean-field model tell us about the dynamics of the cortex?
}

\author{
M.T. Wilson, M.L. Steyn-Ross, D.A. Steyn-Ross, J.W. Sleigh, I.P. Gillies, and \\ D.J. Hailstone
}

\subsection{Introduction}

In this chapter we consider the dynamics of the cortex. We will show that meanfield models can be applied to predict and explain large-scale features of the electroencephalogram (EEG), such as seizures and K-complexes. The changes between these states can be viewed as phase transitions. A cortical mean-field model is perhaps well suited for investigating EEG behavior, given that the EEG is a result of sampling large numbers of neurons. The model we consider follows the continuum approach introduced by Nunez [12] and Freeman [2]. This style of model has been developed by, amongst others, Wright and Liley [23], Robinson et al. [16], Liley et al. [9], and Rennie et al. [14]. Here we implement a two-dimensional model in the manner of Liley et al. [9], that specifically incorporates the dynamics of neuron somas, synapses and axonal propagation.

Mean-field models have a history of being used to describe phase transitionsfor example, the Weiss model of ferromagnetism. Although it is known that meanfield models are lacking in their description of correlations (and these can influence the critical exponents of second-order phase transitions), such a basis is a natural place to begin for describing phase transitions in the cortex. In the limit of spatially symmetric perturbations, the model exhibits stable and unstable nodes, and undergoes Hopf bifurcations into limit cycles. We identify these limit cycles as seizures. If the symmetry is relaxed, traveling-wave solutions can be excited, reminiscent of the slow-waves and K-complexes found in sleep. These solutions are related to the

\footnotetext{
Marcus T. Wilson - Moira L. Steyn-Ross - D. Alistair Steyn-Ross · Iain-Patrick Gillies · David J. Hailstone

Dept of Engineering, University of Waikato, Private Bag 3105, Hamilton 3240, New Zealand.

e-mail:m.wilson@waikato.ac.nz msr@waikato.ac.nz asr@waikato.ac.nz

Jamie W. Sleigh

Waikato Clinical School, University of Auckland, Waikato Hospital, Hamilton 3204, New Zealand. e-mail: sleighj@waikatodhb.govt.nz
} 
spatially symmetric limit cycles. Also, spiral-wave solutions have been found from numerical simulation.

\subsection{A mean-field model of the cortex}

The cortex consists of a large number of macrocolumns, each containing around $10^{5}$ neurons in a volume of $\sim 1 \mathrm{~mm}^{2}$ area by $\sim 1 \mathrm{~mm}$ thickness. We model the cortex as a two-dimensional continuous area of macrocolumns. We follow the mean-field continuum approach of Liley et al. [9] in using a set of coupled differential equations in time and space to describe the excitatory and inhibitory soma potentials $V_{e}$ and $V_{i}$ within the cortex, and the time-evolution of postsynaptic potentials (PSPs). This approach, in which the postsynaptic fluxes $\Phi_{j k}$ (where $j$ and $k$ can correspond to excitatory $e$ or inhibitory $i$ neuron populations) are described by differential equations, is equivalent to approaches that describe the build-up of potentials in terms of time-integrated inputs, for example that of Jirsa and Haken [6]. We use a standard wave-equation, in the manner of Robinson et al. [16], to describe the propagation of presynaptic fluxes $\phi_{j k}$ from one part of the cortex to another. We model the subcortical input with white noise.

It is important to note that individual firing events are not modeled explicitly, instead the effects of a firing of a population of neurons are considered.

The complete set of equations describing the macrocolumn averages of soma potential and synaptic fluxes, as a function of space and time, are described in Refs [18], [19], and [22], and are given below. Note that later, Eqs (10.1) and (10.2) will be modified slightly by the introduction of neuromodulators into the model.

$$
\begin{gathered}
\tau_{e} \frac{d V_{e}}{d t}=V_{e}^{\mathrm{rest}}-V_{e}+\rho_{e} \psi_{e e} \Phi_{e e}+\rho_{i} \psi_{i e} \Phi_{i e} \\
\tau_{i} \frac{d V_{i}}{d t}=V_{i}^{\mathrm{rest}}-V_{i}+\rho_{e} \psi_{e i} \Phi_{e i}+\rho_{i} \psi_{i i} \Phi_{i i} \\
\left(\frac{d^{2}}{d t^{2}}+2 \gamma_{e e} \frac{d}{d t}+\gamma_{e e}^{2}\right) \Phi_{e e}=\gamma_{e e}^{2}\left(N_{e e}^{\alpha} \phi_{e e}+N_{e e}^{\beta} Q_{e}+\phi_{e e}^{\mathrm{sc}}\right), \\
\left(\frac{d^{2}}{d t^{2}}+2 \gamma_{e i} \frac{d}{d t}+\gamma_{e i}^{2}\right) \Phi_{e i}=\gamma_{e i}^{2}\left(N_{e i}^{\alpha} \phi_{e i}+N_{e i}^{\beta} Q_{e}+\phi_{e i}^{\mathrm{sc}}\right) \\
\left(\frac{d^{2}}{d t^{2}}+2 \gamma_{i e} \frac{d}{d t}+\gamma_{i e}^{2}\right) \Phi_{i e}=\gamma_{i e}^{2}\left(N_{i e}^{\beta} Q_{i}+\phi_{i e}^{\mathrm{sc}}\right) \\
\left(\frac{d^{2}}{d t^{2}}+2 \gamma_{i i} \frac{d}{d t}+\gamma_{i i}^{2}\right) \Phi_{i i}=\gamma_{i i}^{2}\left(N_{i i}^{\beta} Q_{i}+\phi_{i i}^{\mathrm{sc}}\right) \\
\left(\frac{\partial^{2}}{\partial t^{2}}+2 v \Lambda_{e e} \frac{\partial}{\partial t}+v^{2} \Lambda_{e e}^{2}-v^{2} \nabla^{2}\right) \phi_{e e}=v^{2} \Lambda_{e e}^{2} Q_{e}
\end{gathered}
$$




$$
\left(\frac{\partial^{2}}{\partial t^{2}}+2 v \Lambda_{e i} \frac{\partial}{\partial t}+v^{2} \Lambda_{e i}^{2}-v^{2} \nabla^{2}\right) \phi_{e i}=v^{2} \Lambda_{e i}^{2} Q_{e}
$$

In these equations, $V_{e}^{\text {rest }}$ and $V_{i}^{\text {rest }}$ are the excitatory and inhibitory neuron resting potentials, and $\rho_{e}$ and $\rho_{i}$ are the strengths of the EPSP and IPSP response functions (i.e., the area of the plot of PSP response function against time). Note that the inhibitory effect is modeled with a negative $\rho_{i}$. The variables $\psi_{e e}, \psi_{e i}, \psi_{i e}$ and $\psi_{i i}$ are weighting functions dependent upon the soma potentials. They are given by:

$$
\psi_{a b}=\frac{V_{a}^{\mathrm{rev}}-V_{b}}{V_{a}^{\mathrm{rev}}-V_{b}^{\mathrm{rest}}} .
$$

Here, $V_{a}^{\text {rev }}$ is the reversal potential of the type $a$ synapse, due to the concentrations of the neurotransmitters AMPA and GABA. The suffices $a$ and $b$ can take on the labels $e$ and $i$.

The terms $\tau_{e}$ and $\tau_{i}$ describe the time-constants for the $e$ and $i$ neurons. The $\gamma_{a b}$ terms are synaptic rate-constants; their reciprocals give the time-scales over which the EPSPs and IPSPs occur. The mean axonal velocity for long-range interactions is given by $v$, and the characteristic length for long-range interactions is given by $1 / \Lambda_{e a}$. Short-range interactions are not modeled with axonal propagation but are

Table 10.1 The standard parameters for a human cortex used throughout this chapter, except where stated otherwise. In this table the suffix $a$ can take on the labels $e$ and $i$. The values are taken mostly from the paper of Rennie et al. [14]. Although there is considerable uncertainty in these parameters, they form a plausible set that is sufficient for the purposes of elucidating much of the physics of the cortical model. It is quite possible that further physical effects can be produced by varying these parameters sufficiently.

\begin{tabular}{cll}
\hline Parameter & Description & Standard value \\
\hline$\tau_{e, i}$ & membrane time constants & $0.04,0.04 \mathrm{~s}^{-1}$ \\
$Q_{e, i}$ & maximum firing rates & $30,60 \mathrm{~s}^{-1}$ \\
$\theta_{e, i}$ & sigmoid thresholds & $-58.5,-58.5 \mathrm{mV}$ \\
$\sigma_{e, i}$ & standard deviation for threshold & $4.0,6.0 \mathrm{mV}$ \\
$\rho_{e, i}$ & gain per synapse at resting voltage & $0.001,-0.00105 \mathrm{mV} \cdot \mathrm{s}$ \\
$V_{e, i}^{\text {rev }}$ & reversal potentials at synapse & $0,-70 \mathrm{mV}$ \\
$V_{e, i}^{\text {rest }}$ & cell resting potential & $-64,-64 \mathrm{mV}$ \\
$N_{e a}^{\alpha}$ & long-range $e$ to $e$ or $i$ connectivity & 3710 \\
$N_{e a}^{\beta}$ & short-range $e$ to $e$ or $i$ connectivity & 410 \\
$N_{i a}^{\beta}$ & short-range $i$ to $e$ or $i$ connectivity & 800 \\
$\left\langle\phi_{e a}^{\text {sc }}\right\rangle$ & mean $e$ to $e$ or $i$ subcortical flux & $750 \mathrm{~s}^{-1}$ \\
$\left\langle\phi_{i a}^{\text {sc }}\right\rangle$ & mean $i$ to $e$ or $i$ subcortical flux & $1500 \mathrm{~s}^{-1}$ \\
$\gamma_{e a}$ & excitatory synaptic rate constant & $300 \mathrm{~s}^{-1}$ \\
$\gamma_{i a}$ & inhibitory synaptic rate constant & $65 \mathrm{~s}^{-1}$ \\
$L_{x, y}$ & spatial length of cortex in model & $500 \mathrm{~mm}^{2}$ \\
$a_{\mathrm{mc}}$ & area of macrocolumn & $1 \mathrm{~mm}^{2}$ \\
$\Lambda_{e a}$ & characteristic inverse length-scale for connections & $0.2 \mathrm{~mm}^{-1}$ \\
$v$ & mean axonal conduction speed & $1400 \mathrm{~mm} \mathrm{~s}^{-1}$ \\
\hline
\end{tabular}

Page:3 job:Ch10_Wilson macro:svmult.cls date/time: 8-Jul-2009/0:30 
assumed to be instantaneous; the $N_{j k}^{\beta}$ terms couple directly with the population firing rates $Q_{k}$ in equations (10.3)-(10.6).

The sigmoidal functions $Q_{e}$ and $Q_{i}$, describing the population firing-rate of neurons, are given by:

$$
\begin{aligned}
Q_{e}\left(V_{e}\right) & =\frac{Q_{e}^{\max }}{1+\exp \left[-\pi\left(V_{e}-\theta_{e}\right) / \sqrt{3} \sigma_{e}\right]}, \\
Q_{i}\left(V_{i}\right) & =\frac{Q_{i}^{\max }}{1+\exp \left[-\pi\left(V_{i}-\theta_{i}\right) / \sqrt{3} \sigma_{i}\right]} .
\end{aligned}
$$

Here we have introduced further parameters $Q_{e}^{\max }$ and $Q_{i}^{\max }$, the maximum firing rates for the excitatory and inhibitory neurons respectively; $\theta_{e}$ and $\theta_{i}$, the inflexionpoint voltage; and $\sigma_{e}$ and $\sigma_{i}$, the standard deviation of the threshold potential.

Finally, the $N_{a b}^{\beta}$ represent numbers of local intra-macrocolumn connections from type $a$ neurons to type $b$ (again $a$ and $b$ can take on the labels $e$ and $i$ ), and the $N_{e a}^{\alpha}$ represent the number of long-range connections from type $e$ neurons to type $a$ (note that inhibitory neurons have no long-range projections). Noise enters the model through the $\phi_{a b}^{\mathrm{sc}}$ terms.

The list of standard parameters used for a human cortex is given in Table 10.1.

We now modify the equations by introducing two parameters to describe the effects of neuromodulators. Following Steyn-Ross et al. [18], we introduce a term $\Delta V_{e}^{\text {rest }}$ to the excitatory resting potential in Eq. (10.1) to model the archetypal somnogen adenosine, and a multiplier $\lambda$ to the excitatory synaptic strength to account for the effect of acetylcholine (abundant in rapid-eye-movement (REM) sleep, but absent in slow-wave sleep). Equations (10.1) and (10.2) now become:

$$
\begin{gathered}
\tau_{e} \frac{d V_{e}}{d t}=V_{e}^{\mathrm{rest}}+\Delta V_{e}^{\mathrm{rest}}-V_{e}+\lambda \rho_{e} \psi_{e e} \Phi_{e e}+\rho_{i} \psi_{i e} \Phi_{i e} \\
\tau_{i} \frac{d V_{i}}{d t}=V_{i}^{\mathrm{rest}}-V_{i}+\lambda \rho_{e} \psi_{e i} \Phi_{e i}+\rho_{i} \psi_{i i} \Phi_{i i}
\end{gathered}
$$

\subsection{Stationary states}

The full two-dimensional dynamics of the model will be explored by simulation; however, it is instructive to calculate the stationary (equilibrium) states of the model (i.e., solving for the state variables $V_{j}, \Phi_{j k}, \phi_{e j}$, where $j, k=e$ or $i$ ) such that their time- and spatial-derivatives are zero. The solutions are computed numerically as a function of the two parameters $\Delta V_{e}^{\text {rest }}$ and $\lambda$. Figure 10.1 is a plot of the solution for $V_{e}$ against these two parameters (we could also construct associated plots for $V_{i}$, $\Phi_{e e}$, etc). It is immediately clear that there exists a region where there are multiple stationary states. This is suggestive of the presence of phase transitions-e.g., in

Page: 4 job:Ch10 Wilson macro: svmult.cls date/time: 8-Jul-2009/0:30 
Fig. 10.1 Plot of $V_{e}$ for stationary states in the sleep domain, using Table 10.1 parameter values. Multiple stationary states exist in a region of the sleep domain at negative $\Delta V_{e}^{\text {rest }}$. The thickgreen curve marks turning points (where the gradient is infinite). (Modified from Ref. [18]).

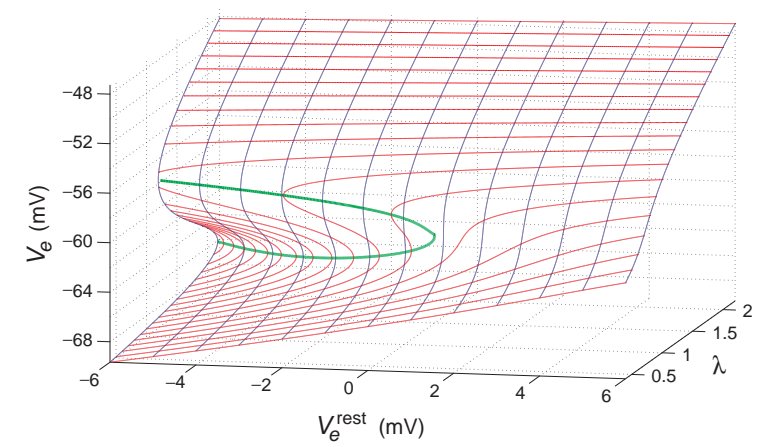

Ref. [18], Steyn-Ross et al. identified the lower (more negative $V_{e}$ ) branch as slowwave sleep (SWS) and the upper branch as REM sleep.

\subsection{Hopf bifurcations}

In this section we investigate the stability of the stationary solutions. Specifically, we will show the presence of Hopf bifurcations that lead to instabilities in the stationary states. Where there is no stable stationary state, a stable spatially-symmetric limitcycle oscillatory state exists. The dynamic solutions to the equations are presented in the next section.

\subsubsection{Stability analysis}

To carry out a stability analysis, we decompose the six second-order equations (Eqs. (10.3)-(10.8)) into pairs of first-order equations, which, along with Eqs. (10.12) and (10.13), give a total of fourteen coupled first-order differential equations in time. Then we perform a first-order series expansion in time about the stationary state. We define uniquely any state of the system by its 14-dimensional state-vector $\mathbf{y}(\mathbf{r})$, which contains the variables $V_{e}, V_{i}, \Phi_{e e}, d \Phi_{e e} / d t, \Phi_{e i}, d \Phi_{e i} / d t, \Phi_{i e}, d \Phi_{i e} / d t$, $\Phi_{i i}, d \Phi_{i i} / d t, \phi_{e e}, d \phi_{e e} / d t, \phi_{e i}$, and $d \phi_{e i} / d t$. These are all functions of space $(\mathbf{r})$, and Eqs. (10.3)-(10.8), (10.12) and (10.13) describe explicitly how these evolve with time $t$. We then assume a small plane-wave perturbation of the system in twodimensional space of wave vector $\mathbf{q}$, about the stationary point $\mathbf{y}_{\text {eqm }}$. That is,

$$
\mathbf{y}(\mathbf{r})=\mathbf{y}_{\text {eqm }}+\Delta \mathbf{y}^{\mathbf{q}} \exp (i \mathbf{q . r}),
$$

where the dynamics can be written as a simple matrix equation 


$$
\frac{d}{d t}\left(\Delta \mathbf{y}^{\mathbf{q}}\right)=A(q) \Delta \mathbf{y}^{\mathbf{q}} .
$$

Here, $A$ is a sparse $14 \times 14$ matrix which contains all the dynamics of the system; it is dependent upon the modulus of the wave vector $q=|\mathbf{q}|$. The vector $\Delta \mathbf{y}^{\mathbf{q}}$ denotes the amplitude of the plane-wave perturbation.

In order for the system to be stable to small perturbations about a given stationary point, we require the real parts of all the eigenvalues of $A$ to be negative, for all $q$. We do not present the matrix $A$ here, but have described the method in more detail in Ref. [22].

The choice of wave vector $q$ is important, since some eigenvalues are particularly sensitive to $q$. In all cases, we find that increasing $q$ decreases the real part of the eigenvalues (i.e., makes them less positive or more negative). The perturbations that are most likely to lead to instabilities are therefore those with $q=0$, corresponding to spatially symmetric "breathing" modes. This observation agrees with that made by Robinson et al. using a different model [15], and is confirmed by simulation. In what follows, we assess the stability of the system by constructing the matrix $A(q=0)$, then finding its eigenvalues. If one or more eigenvalues has a positive real part, then Eq. (10.15) predicts an instability.

\subsubsection{Stability of the stationary states}

We begin by presenting a typical result for the stability of the sleep domain.

Figure 10.1 showed graphically the stationary values for $V_{e}$ : across most of the domain, there is just one stationary state; however, for a small region there are three stationary states. Figure 10.2 shows the stability of these states. There is a small lake-like region where the linearized system has a pair of eigenvalues with positive real part, corresponding to an instability. The nature of this region is discussed in Ref. [22]; notably the edge of the lake-like region corresponds to a supercritical Hopf bifurcation. The real parts of these eigenvalues are most positive when $q=0$, and reduce monotonically as $q$ increases.

Let us now comment on how the situation changes with a different parameter set. We find that the size of the unstable "lake" of Fig. 10.2 is particularly dependent on the choice of $\gamma_{i}$ (we assume $\gamma_{i e}=\gamma_{i i}$, so we can use a single index without ambiguity). This is to be expected since it is this term that governs the rate at which negative (stabilizing) feedback is applied to the system. A small $\gamma_{i}$ corresponds to an IPSP that is spread-out in time; this allows positive feedback from the excitatoryexcitatory route to build up before being quenched. Figure 10.3 shows the stability of the domain when $\gamma_{i}$ is reduced from $65 \mathrm{~s}^{-1}$ to $15 \mathrm{~s}^{-1}$. The stationary solutions remain unchanged; however, the unstable lake has now grown into the the area of three stationary states. This means that either or both of the upper and lower branches, in addition to the mid-branch, can become unstable. There is the possibility of the system having multiple stationary states, with none of them being stable.

Page:6 job:Ch10_Wilson macro:svmult.cls date/time:8-Jul-2009/0:30 
Fig. 10.2 Plot of the sleep domain showing a region of multiple stationary states (shaded area to upper-left), and a region of single, unstable stationary states (shaded "lake" ), for the parameters of Table 10.1. The system is stable in the unshaded regions (e.g., at the point marked $\ominus$ ), and unstable at the point marked $\oplus$. (Reprinted from [22] with permission.)

Fig. 10.3 Stability of the sleep domain for a decrease in $\gamma_{i}$ to $15 \mathrm{~s}^{-1}$. The unstable lake on the right has grown into the region of multiple stationary states. Five possibilities exist: one stable state; one unstable state; three unstable states (e.g., at point $\oplus$ ); one stable state and two unstable; two stable states and one unstable. (The mid-branch state is always unstable.) The unmarked region of the figure corresponds to a single, stable state. (Reprinted from [22] with permission.)
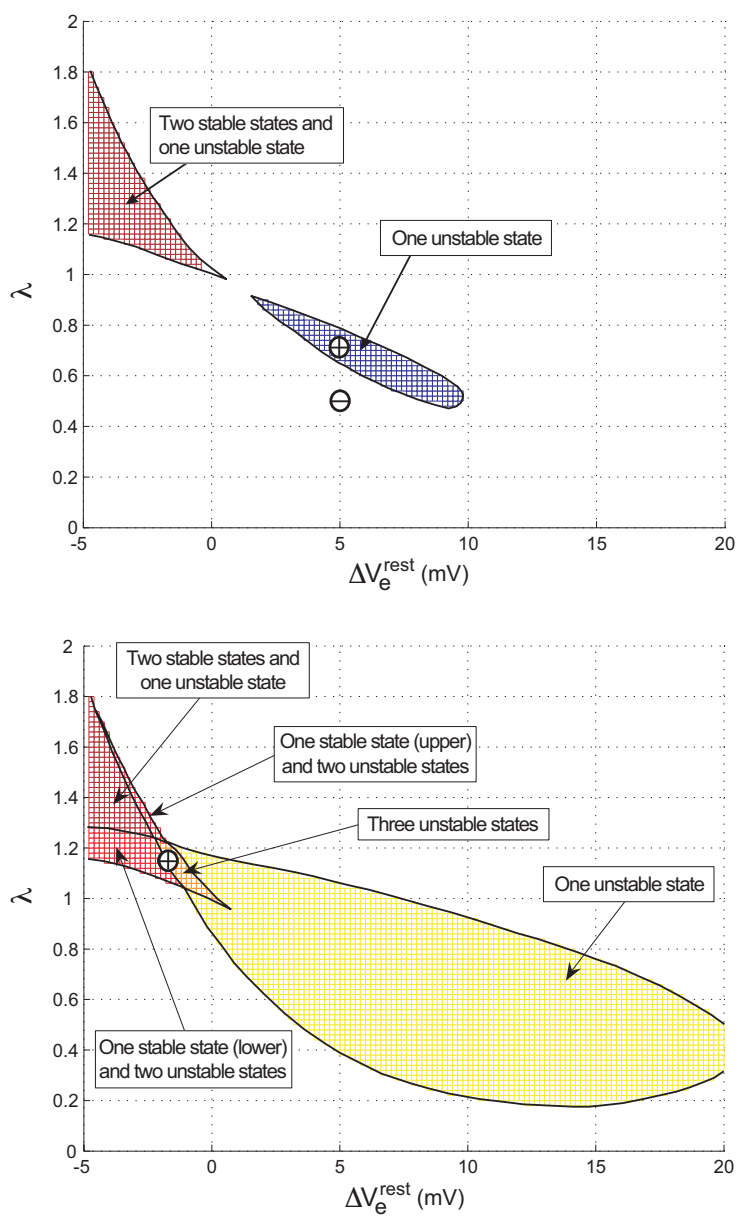

The limit cycles associated with these instabilities are found by simulation, and are described in the next section.

\subsection{Dynamic simulations}

Numerical simulations of the equations on a two-dimensional grid allow the exploration of a range of different dynamic behaviors. We first outline how this is done, then consider spatially symmetric and asymmetric solutions. We tentatively suggest how these solutions might correspond with gross features of the EEG. The parameter values used here are broadly representative of human cortex; parameters and 
bifurcation structures more appropriate for rat cortex are discussed in the chapter by Sleigh et al. ${ }^{1}$

In one dimension, a study of spatial and temporal structures such as traveling waves in a similar model has been performed by Hutt et al. [5] using both a stability analysis similar to that in Sect. 10.4.1, and numerical simulations.

We have performed grid simulations on the full set of equations in 2-D space. We used a regular square grid to represent the cortex in space, and iterated the full equations in time using an order-1 Euler predictor-corrector method [7], as detailed in reference [22]. Noise is fed into the system through the subcortical terms, but does not affect gross dynamic behaviors that dominate when there is no stable stationary state available.

\subsubsection{Breathing modes}

We now look at the stability and dynamics of the system in more detail. We consider vertical slices across the domains of Figs. 10.2 and 10.3: we fix $\Delta V_{e}^{\text {rest }}$, and show the mean soma potential as a function of $\lambda$. The slice-plot for Fig. 10.2 (with $\left.\gamma_{i}=65 \mathrm{~s}^{-1}\right)$ is shown in Fig. 10.4; and the slice-plot for Fig. $10.3\left(\gamma_{i}=15 \mathrm{~s}^{-1}\right)$ is shown in Fig. 10.5. Also indicated on these plots is the stability of the solution (solid = stable, dashed $=$ unstable), and the extent of "breathing mode" (spatially symmetric) limit cycles. The breathing-mode cycle, corresponding to $q=0$, is always the most unstable perturbation, and is usually the cycle reached in the limit of large times. The dominance of the $q=0$ instability arises because the model used in this chapter has no spatial diffusion; when diffusive coupling in space is introduced-for example, via gap-junctions-instabilities at nonzero $q$ can dominate, as discussed in the chapter by Steyn-Ross et al. ${ }^{2}$

The limit cycles were found by simulation, and are indicated by plotting thicksolid lines in Figs 10.4 and 10.5 to show the maximum and minimum values of the mean excitatory soma potential during the cycle; unstable limit cycles associated with these Hopf bifurcations are not shown.

We start with Fig. $10.4\left(\gamma_{i}=65 \mathrm{~s}^{-1}\right)$, and discuss the solutions with reference to stepped decreases in the $\Delta V_{e}^{\text {rest }}$ parameter. For $\Delta V_{e}^{\text {rest }}$ larger than about $+10 \mathrm{mV}$, there is a single stationary state, and this is stable (e.g., see Fig. 10.4(a)). When $\Delta V_{e}^{\text {rest }}$ is reduced, a small unstable region appears, associated with a pair of Hopf bifurcations (marked with " $\times$ " on Fig. 10.4(b, c, d)). If a simulation is performed in the unstable region, a spatially-symmetric (breathing mode) limit cycle results, with an amplitude extent as marked on the plot with thick-solid lines. Note the supercritical nature of the Hopf bifurcations for this value of $\gamma_{i}$.

This region of instability disappears when $\Delta V_{e}^{\text {rest }}$ is reduced to about $1 \mathrm{mV}$. At the same time the slope of membrane potential against $\lambda$ increases; and for $\Delta V_{e}^{\text {rest }}$

\footnotetext{
${ }^{1}$ See Ch. 9 of the present volume.
}

${ }^{2}$ See Ch. 12 of the present volume.

Page: 8 job:Ch10_Wilson macro: svmult.cls date/time: 8-Jul-2009/0:30 

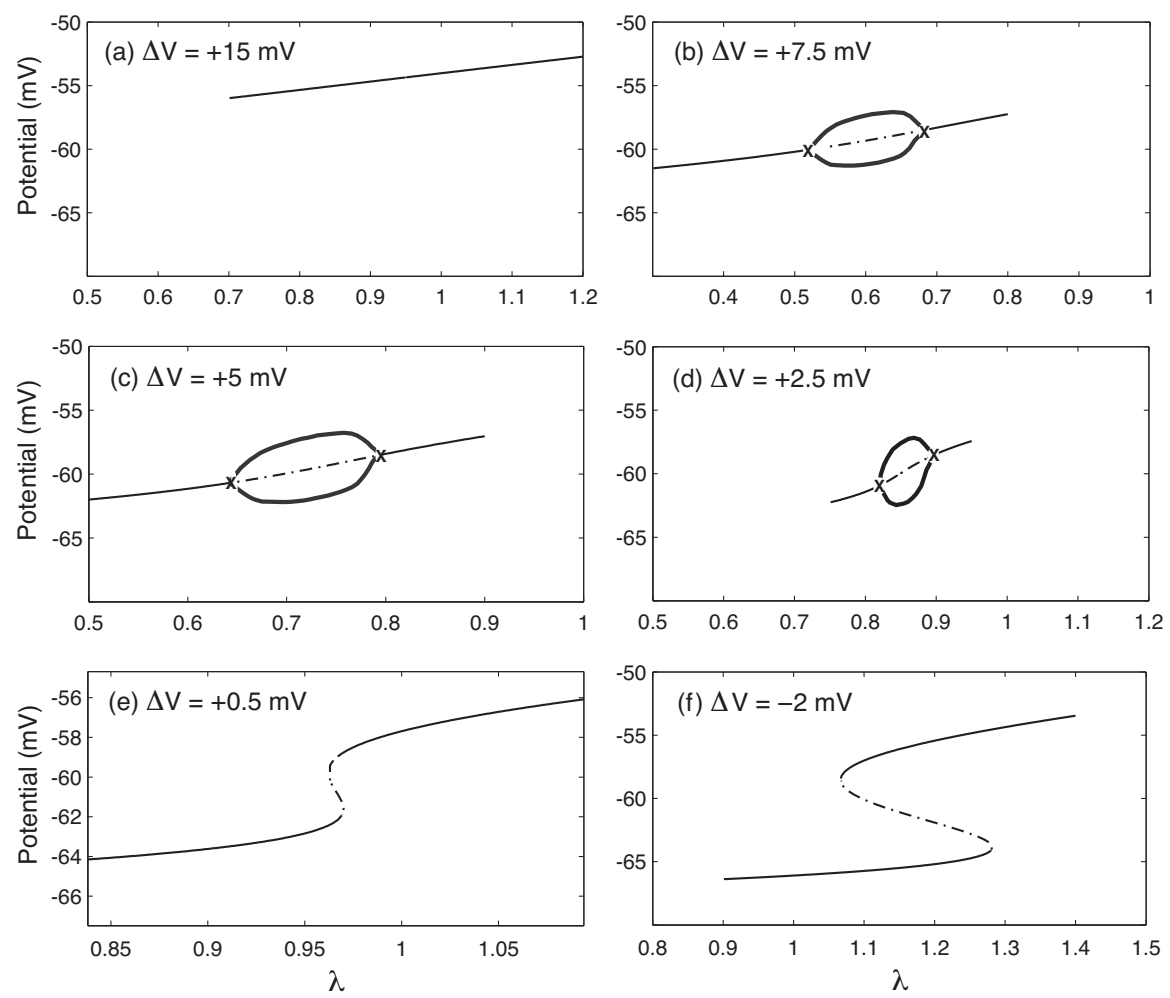

Fig. 10.4 Stationary states, bifurcations (found by calculation), and breathing modes (found by simulation) corresponding to six vertical slices through Fig. 10.2 with $\gamma_{i}=65 \mathrm{~s}^{-1}$. Graphs plot excitatory soma potential $V_{e}$ versus excitatory synaptic strength $\lambda$ for six values of $\Delta V_{e}^{\text {rest }}$ (displayed in the subtitles as $\Delta V$ ). Thin line indicates stationary states of $V_{e}:$ solid = stable; dotted $=$ unstable. Supercritical Hopf bifurcations are marked with $\times$. Thick-solid line denotes maximum and minimum extent of the associated limit cycle (only stable part of the limit cycle is shown).

less than about $1 \mathrm{mV}$ (see Fig. 10.4(e, f)), multiple stationary states can exist (i.e., there exists a saddle-node bifurcation for variations in $\lambda$.) The middle branch of the three solutions is unstable. As $\Delta V_{e}^{\text {rest }}$ decreases further, the extent of the multiple solutions increases.

We now look at the case of a time-lengthened IPSP, where $\gamma_{i}=15 \mathrm{~s}^{-1}$. Figure 10.3 shows that this delayed feedback leads to a large increase in instability; the corresponding slice-plots are shown in Fig. 10.5. There are three key differences from Fig. 10.4. First, we note that the extent of the unstable area (the region between Hopf bifurcation pairs) has increased. Second, the bifurcations have become subcritical-i.e., the limit cycle extends beyond the region bracketed by the Hopf bifurcations. This means that the system can have both a stable node and a stable limit cycle available to it simultaneously. Finally, we see that the unstable region co-exists with the region of multiple stationary state solutions. This can result in a rich variety of solutions; for example, at $\Delta V_{e}^{\text {rest }}=-2.5 \mathrm{mV}$ and $\lambda=1.25$ there 

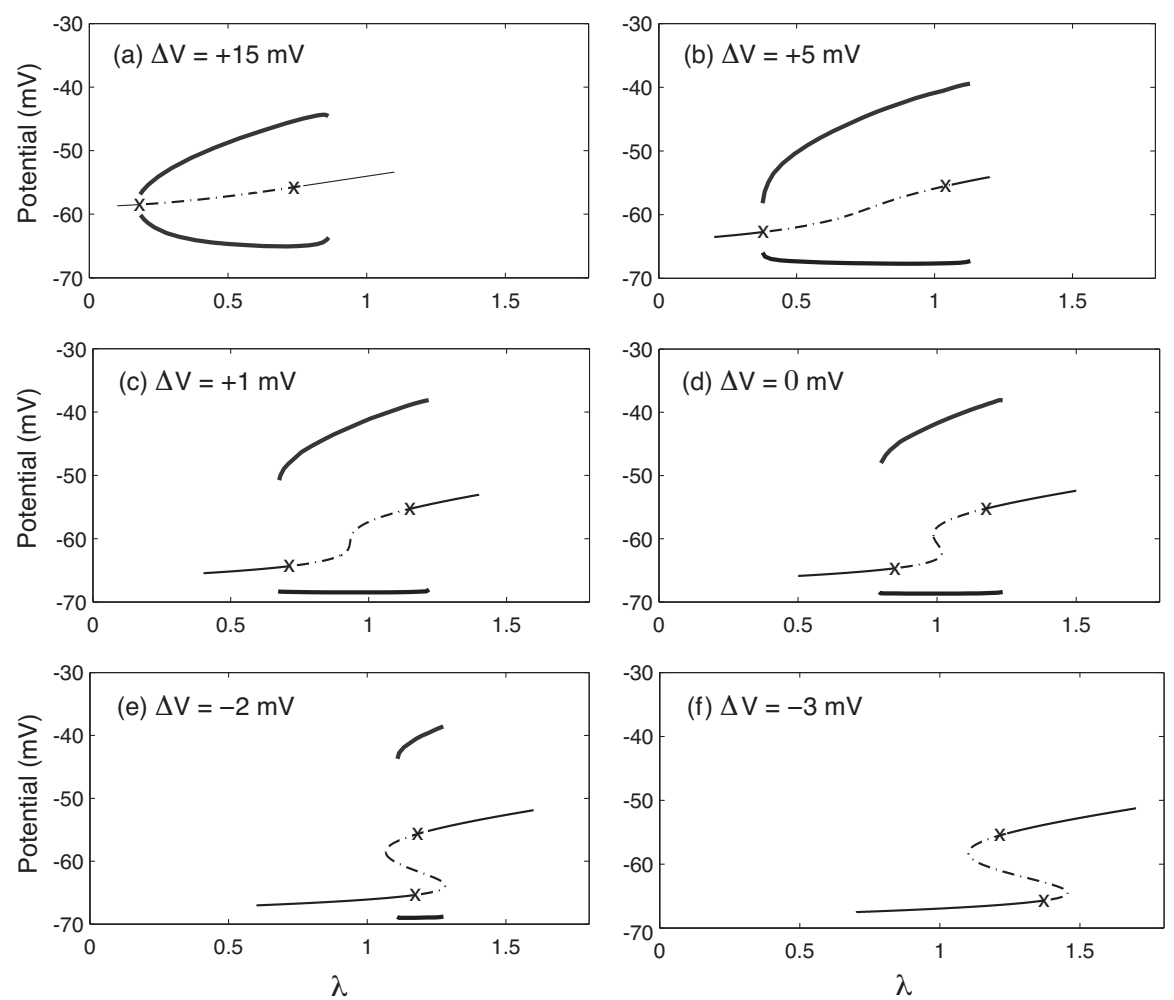

Fig. 10.5 Stationary states, bifurcations, and breathing modes corresponding to six vertical slices through Fig. 10.3: $\gamma_{i}$ has been reduced to $15 \mathrm{~s}^{-1}$. Graphs plot $V_{e}$ versus $\lambda$ for six values of $\Delta V_{e}^{\text {rest }}$ (displayed in the subtitles as $\Delta V$ ). Thin line indicates stationary states of $V_{e}$ : solid = stable; dotted $=$ unstable. Subcritical Hopf bifurcations are marked with $\times$. Thick-solid line denotes maximum and minimum extent of the associated stable limit cycle.

are two stable stationary states and a stable limit cycle available (see Fig. 10.6). For negative $\Delta V_{e}^{\text {rest }}$, an unstable "lip" remains on the top branch of the stationary states, and a very small unstable lip remains on the bottom branch. (See also Fig. 3).

The Fig. 10.5 limit cycles arise as a result of small inhibitory synaptic rateconstant (i.e., large synaptic time-constant), leading to an effective delay in application of negative feedback. The formation of this instability has been linked with seizures (e.g., Kramer et al. [8]), and in Ref. [20] we have suggested that the tendency for the anesthetic enflurane to promote seizures is due to its lengthening of the inhibitory postsynaptic potential, causing Hopf bifurcation to a limit cycle. It is unlikely that a healthy brain would operate in such a region. 
Fig. 10.6 Stationary states for the excitatory membrane potential $V_{e}$ for the case $\Delta V_{e}^{\text {rest }}=-2.5 \mathrm{mV}, \gamma_{i}=15 \mathrm{~s}^{-1}$. Stable stationary states are marked with a thin-solid line, unstable states with a dot-dashed line. Subcritical Hopf bifurcations are marked with $(\times)$. The limit cycle is indicated by thick-solid lines showing the upper and lower extents of the excitatory membrane potential. Note that for $\lambda \approx 1.25$, the system has access to two stable stationary states and a stable limit cycle.

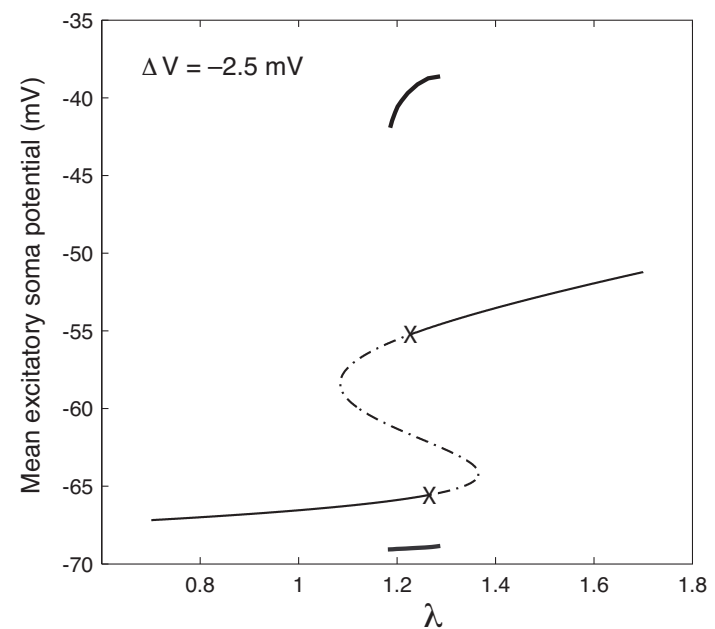

\subsubsection{Response to localized perturbations}

To investigate the dynamic, spatially asymmetric response of this model, we look specifically at the response to small "kicks" to the cortex, which physically could be a result of thalamic pathways to the cortex, or spontaneous neurostransmitter release. As will be shown below, these kicks can produce traveling-wave structures which we will identify with slow waves or K-complexes [21].

We have carried out simulations on a $64 \times 64$ spatial grid, for different values of the control parameter $\lambda$ for the case $\gamma_{i}=15 \mathrm{~s}^{-1}$ and $\Delta V_{e}^{\text {rest }}=-2.5 \mathrm{mV}$. This choice of parameters leads to the stationary-state structure of Fig. 10.6, and gives rich dynamic behavior. In these simulations we place the system initially in a stationary state (where multiple stationary states exist we start on the lower-branch). The noise input results in the soma potential $V_{e}$ undergoing fluctuations about this stationary state, as described by Wilson et al. [22]. Here, we examine the response of the system to a sharp kick applied at a single point in space. This has been done by making a momentary $\left(0.1 \mathrm{~s}\right.$ ) increase in $\Delta V_{e}^{\text {rest }}$ (by $10 \mathrm{mV}$ ) at a single point on the simulation grid (e.g., $\Delta V_{e}^{\text {rest }}$ becomes $+7.5 \mathrm{mV}$ for $0.1 \mathrm{~s}$, before returning to $-2.5 \mathrm{mV}$ ). This corresponds to a large increase in cortical excitation at this point in space only.

With reference to the stationary states shown in Fig. 10.6, for $\gamma_{i}=15 \mathrm{~s}^{-1}$, we will now present results for simulations at various points along the $\lambda$ axis. We start with $\lambda$ small, where there is just one stationary state, and then raise $\lambda$ to move into the region of multiple stationary states, then into the region where the limit cycle exists. Results are presented as grayscale plots (white $=$ high, black $=$ low) of the excitatory soma potential $V_{e}$ for a slice through the cortex (incorporating the position of the kick) against time. We look first at $\lambda=0.8$ where there is only a single, stable stationary state available that is well away from the region of multiple stationary states. Figure 10.7 shows that a kick of $10 \mathrm{mV}$ for $0.1 \mathrm{~s}$ does not lead to 
any interesting dynamics - the soma potential rises at the location of the kick only, and then decays quickly back to the stationary state.

Fig. 10.7 Excitatory soma potential as a function of time in response to a short, localized impulse when only one stationary state is accessible to the system. Parameter values: $\Delta V_{e}^{\text {rest }}=-2.5 \mathrm{mV}$, $\gamma_{i}=15 \mathrm{~s}^{-1}$, with $\lambda=0.8$. The effect of the kick does not propagate. (Reprinted from [21] with permission.)

Fig. 10.8 Excitatory soma potential as a function of time for a short kick at one point in space when lower branch is stable, upper branch unstable. Parameter values: $\Delta V_{e}^{\text {rest }}=$ $-2.5 \mathrm{mV}, \gamma_{i}=15 \mathrm{~s}^{-1}$, with $\lambda=1$.1. Left-hand graph shows $V_{e}$ for two points on the cortex (the point of initial kick, and $25 \mathrm{~cm}$ from this point) versus time. Righthand image shows $V_{e}$ as grayscale (white $=$ high, black = low) against space and time for a line through the position of the kick. The resulting traveling wave is similar to a K-complex. (Reprinted from [21] with permission.)
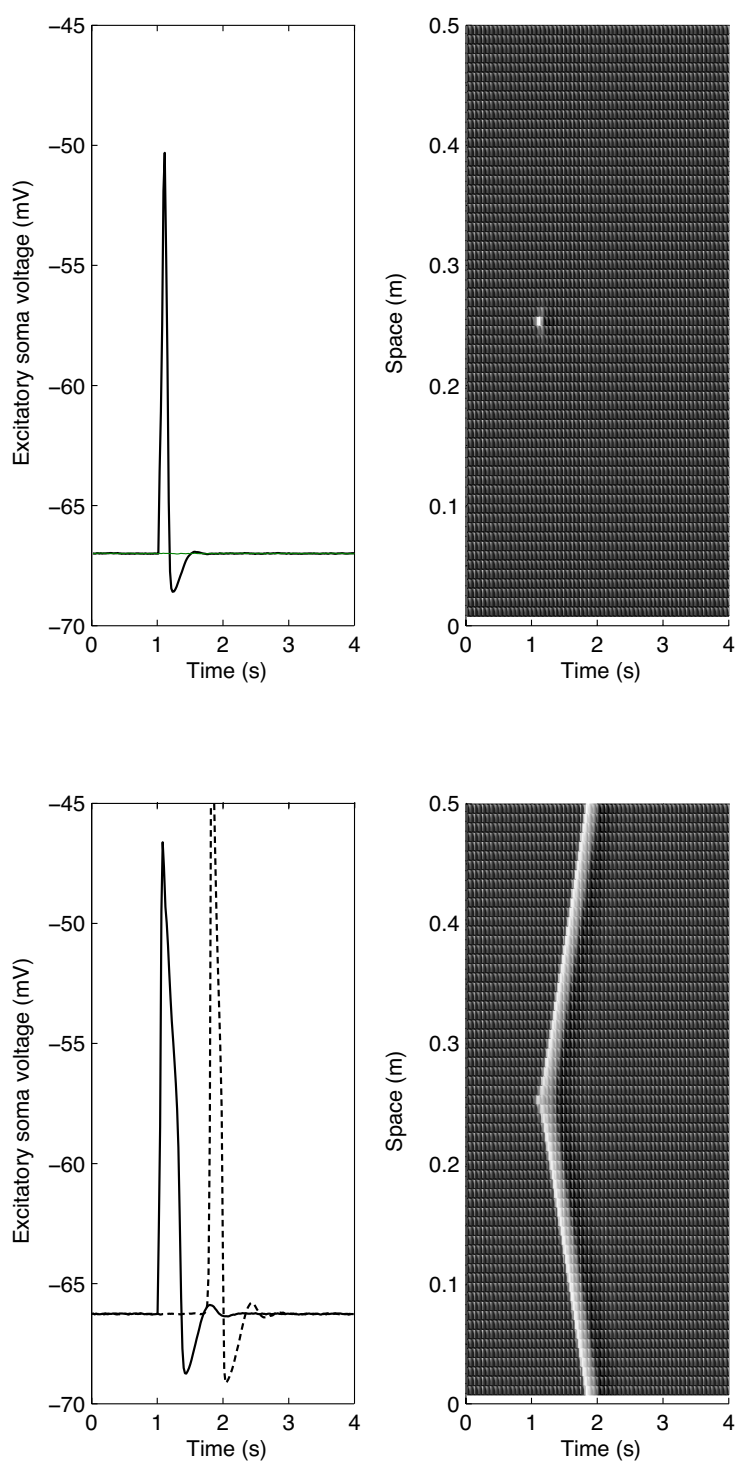

However, the dynamics changes markedly as we approach the region of multiple stationary states. We look at the response of the system to the same kick, but with the control parameter increased to $\lambda=1.1$; there are now three stationary states, but only the lower-branch state is stable. The results are shown in Fig. 10.8. We see that the response propagates as a wave of constant velocity over the whole cortex. 
The system is pushed from its stable lower branch, beginning at the point where the impulse is applied, and heads towards the unstable upper branch. However, since the upper branch is unstable, it cannot remain there, and instead returns to the stable lower branch, where it finally comes to rest (the kick having been removed). This process generates a single circular traveling wave that propagates outwards from the point of the initial kick-every point in space leaves the lower branch, heads towards the upper branch, overshoots, then falls down towards the lower branch, overshoots this, and eventually settles back on the lower branch. The shape of the plot of Fig. 10.8 is similar to that of the K-complex seen in EEGs. Indeed, we will follow Massimini et al. [10] and identify this traveling wave with a K-complex. Golomb and Amitai have shown that a disinhibited network model can also give a similar result [3] - the significant difference between their work and ours is that inhibitory neurons are included here, and multiple stationary states are involved.

The cause of the K-complex in the EEG is uncertain-it can occur either as a response to a specific stimulus (e.g. a sound), or sometimes spontaneously with no obvious cause. In his summary paper, Colrain [1] describes the K-complex, with reference to the earlier work of Numminen et al. [11] as,

- a nonspecific reaction of the cortex to various stimuli during sleep, via activation of projection pathways from the nonspecific thalamic nuclei.

We now raise $\lambda$ further, to $\lambda=1.25$. From Fig. 10.6 we note that, in addition to the stable stationary state of the lower branch, there is now a stable limit cycle available to the spatially-homogeneous system. When we apply a kick to the system
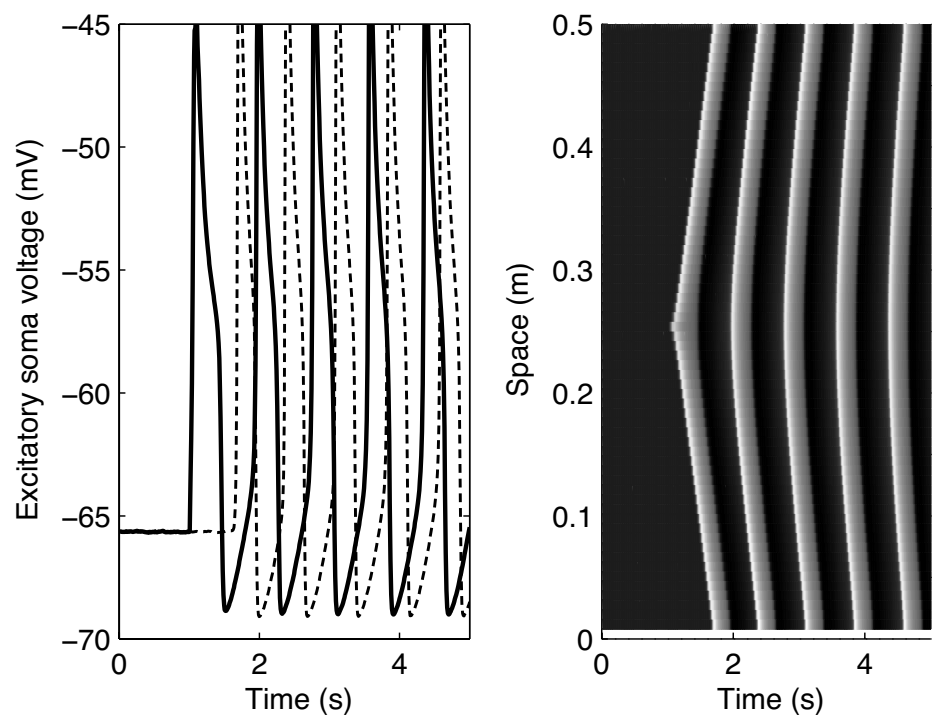

Fig. 10.9 Excitatory soma potential as a function of time when there is a limit cycle available to the system. Parameter settings: $\lambda=1.25, \Delta V_{e}^{\text {rest }}=-2.5 \mathrm{mV}, \gamma_{i}=15 \mathrm{~s}^{-1}$. Note how the limit cycle begins to synchronize in space as time increases. (Reprinted from [21] with permission.)

Page:13 job:Ch10_Wilson macro:svmult.cls date/time: 8-Jul-2009/0:30 
that is initially on the lower branch, the system is provoked into this limit cycle, as illustrated in Fig. 10.9. The firing rate moves from near zero to near maximum, in a similar way to spike-wave seizures [17]. Figure 10.9 also shows the limit cycle becoming progressively more synchronized in space as time increases, corresponding to the $q=0$ breathing mode.

To explore the possibilities further, we look at the crucial significance of the inhibitory synaptic rate-constant, $\gamma_{i}$, in determining the stability of the cortical model. An increase in $\gamma_{i}$ to $65 \mathrm{~s}^{-1}$ leads to a stabilization of the stationary states-i.e., the Hopf bifurcations and limit cycles are removed from Fig. 10.6. In this case a kick produces no traveling waves-i.e., the existence of the traveling wave (K-complex) state depends upon having a sufficiently long inhibitory time-constant.

\subsubsection{K-complex revisited}

We return to the situation that produced the single traveling-wave solution which we identify as a K-complex [21], and explore the response to impulsive kicks of varying size. In Fig. 10.10 we have summarized the four possible dynamic outcomes of a voltage kick of given size at a given value of the parameter $\lambda$. Where there are multiple stationary states, we consider the system to be initially on the lower branch. We set $\Delta V_{e}^{\text {rest }}=-2.5 \mathrm{mV}$ and $\gamma_{i}=15 \mathrm{~s}^{-1}$, i.e., the stability diagram of Fig. 10.6 applies.

At large $\lambda$ (corresponding to $\lambda>1.27$ ), a small kick results in the system moving to the upper state where the stability is greater. Indeed, for $\lambda>1.35$ (region (d) of Fig. 10.10), there is no stable lower state, and the system moves spontaneously to the upper state without any applied kick.

For $1.0<\lambda<1.27$ (region (c)) a kick of sufficient size will result in a traveling wave of the form of Fig. 10.8. Note that the size of the kick is important; if it is not large enough, the disturbance will rapidly die away rather than propagating over space, as in Fig. 10.7. The required size of the kick reduces as $\lambda$ increases; the lower stationary state has become less stable. Note that the boundary between regions (c) and (d) is very distinct.

For $\lambda<1.0$, no kick can generate a traveling wave. However, a large enough kick (region (b) of Fig. 10.10) will result in initial local growth of the disturbance, but one that fails to propagate entirely to other regions. At very low $\lambda$, the boundary between the region of initial growth (b) and of initial decay (a) is quite indistinct; it is marked on Fig. 10.10 by dashed lines. However, the boundary between regions (b) and (c) is very sharp.

This abrupt triggering of a K-complex in region (c) can be explained with reference to the orbits of the spatially-homogeneous system in phase-space. In Fig. 10.11(a), for $\Delta V_{e}^{\text {rest }}=-2.5 \mathrm{mV}, \lambda=1.15$, we plot a projection of the trajectories in phase-space of the spatially-homogeneous system. The top graph is for a small inhibitory synaptic rate-constant, $\gamma_{i}=15 \mathrm{~s}^{-1}$; the bottom graph is for a larger rate of $65 \mathrm{~s}^{-1}$. All state variables have been started at their equilibrium values except

Page: 14 job:Ch10_Wilson macro: svmult.cls date/time:8-Jul-2009/0:30 
for $\Phi_{e e}$ and $\Phi_{i i}$. The equations (10.1-10.8), if written as a set of first-order differential equations, have fourteen dynamic variables; the two variables that illustrate the situation most clearly are $\Phi_{e e}$, the $e \rightarrow e$ synaptic flux, and $\Phi_{i i}$, the $i \rightarrow i$ synaptic flux. For this reason, these two variables have been plotted here. For the top case, since there is only one stable solution, all of the trajectories eventually end on the lower-branch solution (Os in lower left-hand corner). However, looking at the trajectories starting in this vicinity, it is clear that two initial points very close together can generate manifestly different trajectories in order to return to the single stable solution. In one case, the trajectory returns quickly to the stable solution of the lower branch; in the other, first the $e \rightarrow e$ synaptic flux grows, then the $i \rightarrow i$ flux, the $e \rightarrow e$ flux diminishes, and finally the $i \rightarrow i$ flux diminishes, and the trajectory returns to the stable lower branch (Os). This path takes the system around the unstable solution of the upper branch $(\Delta u$ in top-right corner). This divergence of trajectories explains why a tiny increase in the size of the kick (e.g., from region (a) to region (c) of Fig. 10.10) can result in a very different solution to the dynamical equations.

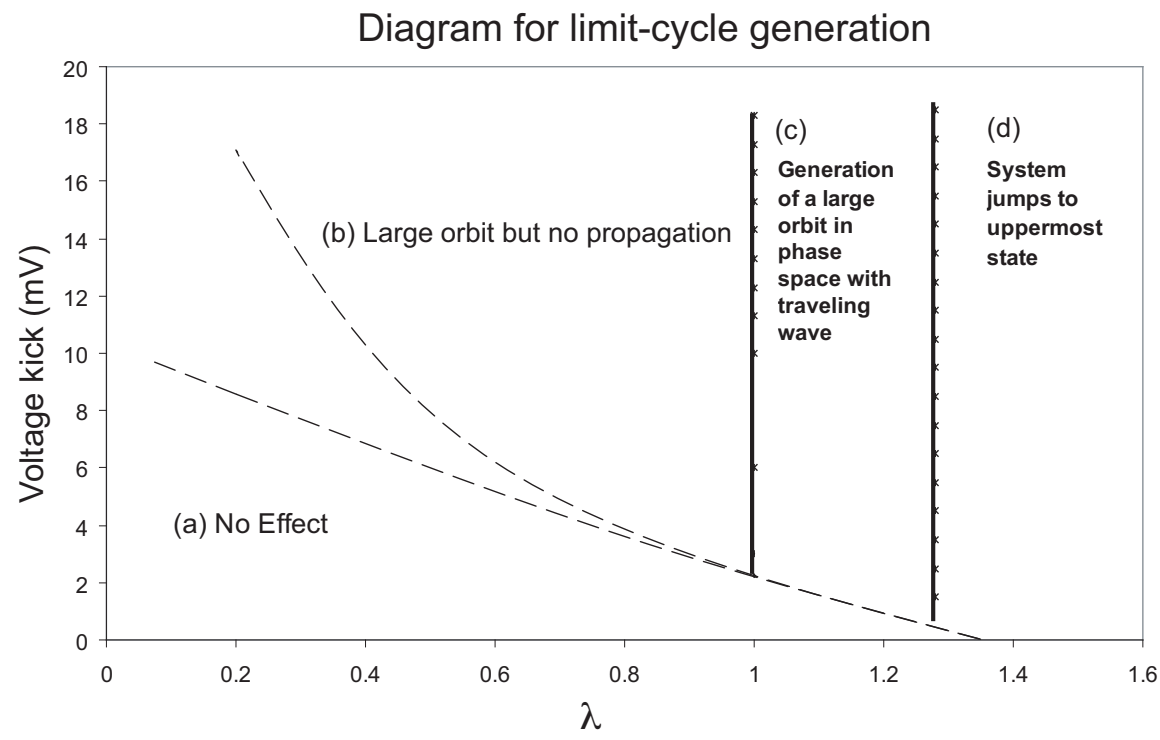

Fig. 10.10 Summary of the effect of disturbing the cortical system at one point in space. Parameter settings: $\Delta V_{e}^{\text {rest }}=-2.5 \mathrm{mV}, \gamma_{i}=15 \mathrm{~s}^{-1}$. (a) For small kicks, except when the lower branch is unstable, the generated disturbance quickly dies away. (b) At low $\lambda$, a large kick can result in a large, localized response (an orbit in phase space similar to Fig. 10.11) but with no propagation away from the site of disturbance. (c) In the vicinity of the region where there are multiple stationary states, with the top state being unstable, a sufficiently-sized kick can generate a large disturbance that propagates as a wave. Note that this can occur even when there is only one stationary state (e.g., $\lambda=1.0$ ); however the system has to be close to the saddle-node bifurcation. The boundary between a (c) propagating and (b) nonpropagating disturbance is very distinct. (d) At high values of $\lambda$, a small kick will displace the system onto the upper state, where it will remain. Again, a distinct boundary separates regions (c) and (d). 

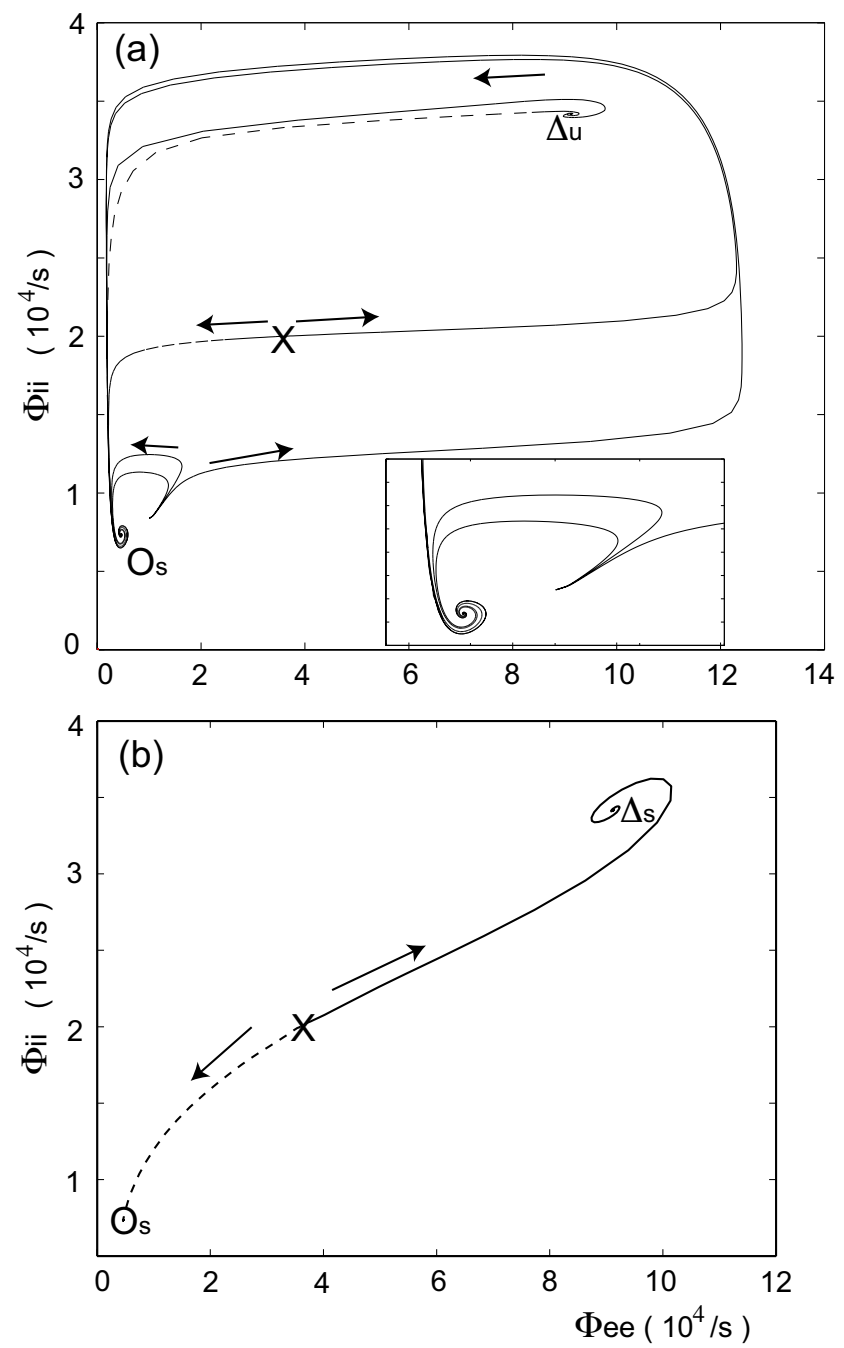

Fig. 10.11 Phase-space trajectories for parameter settings $\Delta V_{e}^{\text {rest }}=-2.5 \mathrm{mV}, \lambda=1.15$, and (a) $\gamma_{i}=15 \mathrm{~s}^{-1}$; (b) $\gamma_{i}=65 \mathrm{~s}^{-1}$. Panel (a): Lower, stable stationary state is in the bottom left-hand corner of the figure (marked "Os"); the unstable upper state in the top-right corner $(\Delta u)$; the unstable mid-branch solution is in the center $(\times)$. Two trajectories have been started on each of the upperand mid-branches - these eventually reach the lower, stable branch (Os). Other trajectories have been started close to the lower state. Two of these return quickly to this state, but the third, initially displaced by a very small distance from the first two, exhibits a markedly different trajectory that loops around the upper state $(\Delta \mathrm{u})$. We identify this trajectory with a K-complex. The vicinity of the lower state has been expanded in the subpanel for clarity (but the different starting points are still indistinguishable). Panel (b): Trajectories have changed markedly with a reduced synaptic response-time. The stationary states remain in the same positions, but the upper state is no longer unstable (now marked " $(\Delta s)$ "). There is no longer divergence of trajectories as in (a). The solid and dotted lines denote different trajectories leaving the same unstable initial point. (Reprinted from [21] with permission.)

Page: 16 job:Ch10_Wilson macro:svmult.cls date/time:8-Jul-2009/0:30 
Why does the numerical simulation produce a traveling wave? Different points in space are coupled through the $\nabla^{2}$-term of Eqs (10.7) and (10.8). If a single point in space is kicked from its stable equilibrium onto the trajectory that travels around the upper branch, as happens in Fig. 10.8, the $\nabla^{2}$ spatial-coupling will pull the neighboring points from their position on the lower stable branch onto this trajectory too. These in turn influence their neighbors, sending out a traveling disturbance. After the wave has passed, all points return to the original, lower-branch stable steady state (Os).

In Fig. 10.11(b) we see the effect of restoring the inhibitory rate-constant to $\gamma_{i}=$ $65 \mathrm{~s}^{-1}$, thereby removing the instability from the upper branch of Fig. 10.6. A large kick, large enough to take the system past the unstable mid-state $(\times)$, will cause the system to move to the upper stationary state $(\Delta s)$. This removes the possibility of producing a K-complex, and instead, a sufficiently large kick, applied to the bottom branch, results in the system moving directly to the top branch.

\subsubsection{Spiral waves}

Finally, we remark on another limit-cycle that is available to an oscillatory system, namely that of spiral waves. These persistent features sometimes can be generated when a simulation is run through an unstable region of the sleep domain. These waves are spatially structured states consisting of pairs of counter-rotating spirals. Figure 10.12 shows a gray-scale plot of $V_{e}(\mathbf{r})$ at a given time. Specifically, this simulation involved starting on a stable, upper-branch solution and then, by reducing $\lambda$, moving the system through an unstable region into the region where the lower branch is stable. These limit-cycles are extremely persistent-to quench them, a large reduction in $\lambda$ or $\Delta V_{e}^{\text {rest }}$ is required (i.e., a large reduction in excitatory component). Experimentally, spirals have been observed in disinhibited cortical slices [4], and demonstrated in neuron models with no inhibition $[4,13]$. However, their presence in the cortex, and their relationship with states such as epileptic seizures, is unclear.

\subsection{Conclusions}

In this chapter we have demonstrated some of the dynamic features associated with a nonlinear mean-field cortical model. In particular, spatially symmetric limit cycles are reminiscent of seizure-like states $[8,20]$, and arise as a result of a delay in negative feedback through a lengthened inhibitory postsynaptic potential. This

delay may explain of the tendancy of some anesthetic drugs, such as enflurane, to promote seizures.

For certain parameter sets, traveling waves of activity can be produced. These are associated with the combination of saddle-node bifurcations and Hopf bifurcations,

Page: 17 job:Ch10_Wilson macro:svmult.cls date/time:8-Jul-2009/0:30 
Fig. 10.12 Snapshot of a spiral wave generated by the cortical model $($ white $=$ high $V_{e} ;$ black $=$ low $\left.V_{e}\right)$. To trigger spiral formation, the system was started on the stable region of the upper branch $\left(\Delta V_{e}^{\text {rest }}=0.5 \mathrm{mV}, \lambda=1.75\right.$, $\left.\gamma_{i}=33 \mathrm{~s}^{-1}\right)$, then $\lambda$ was rapidly lowered (over $\sim 7 \mathrm{~s}$ ) through the unstable region and into the region where only a single stable state exists. Resulting spiral wave is extremely persistent, and requires a considerable further reduction in $\lambda$ to destroy it. (Reprinted from [22] with permission.)

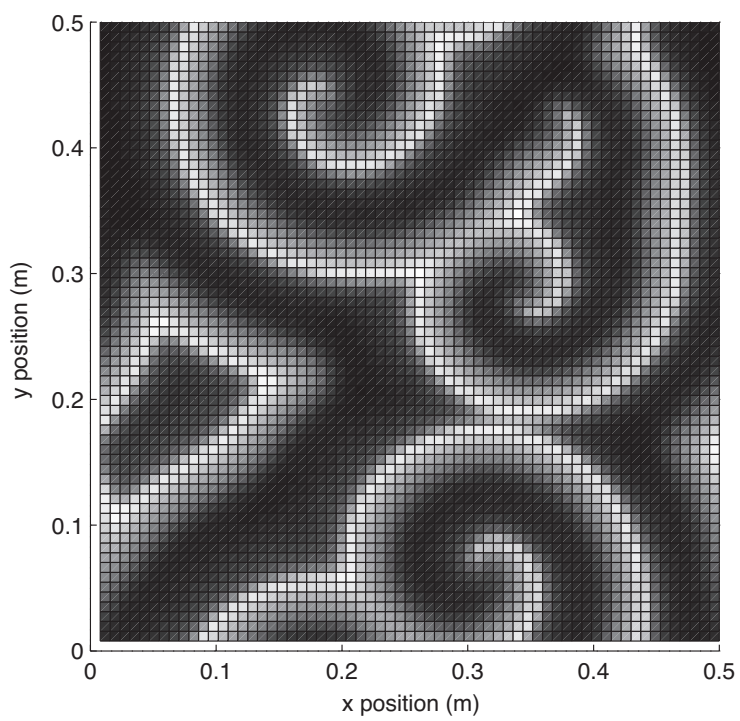

and are reminiscent of the K-complexes and slow oscillations of slow-wave sleep. Such waves can be activated by a point-like disturbance of sufficient magnitude-a below-threshold disturbance will fail to propagate.

In some limited conditions, spiral waves are generated. These are extremely persistent once established. The biological significance of such waves is not entirely clear, although, in general, the ability of a 2-D nonlinear system to produce spiral waves is not surprising.

\section{References}

1. Colrain, I.M.: The K-complex: A 7-decade history. Sleep 28, 255-273 (2006)

2. Freeman, W.J.: Predictions on neocortical dynamics derived from studies in paleocortex. In: E. Basar, T.H. Bullock (eds.), Induced Rhythms of the Brain, chap. 9, pp. 183-199, Birkhaeuser, Boston (1992)

3. Golomb, D., Amitai, Y.: Propagating neuronal discharges in neocortical slices: Computational and experimental study. J. Neurophys. 78, 1199-1211 (1997)

4. Huang, X., Troy, W.C., Yang, Q., Ma, H., Laing, C.R., Schiff, S.J., Wu, J.Y.: Spiral waves in disinhibited mammalian neocortex. J. Neurosci. 24, 9897-9902 (2004), doi: 10.1523/jneurosci.2705-04.2004

5. Hutt, A., Bestehorn, M., Wennekers, T.: Pattern formation in intracortical neuronal fields. Network 14, 351-368 (2003)

6. Jirsa, V.K., Haken, H.: A field theory of electromagnetic brain activity. Phys. Rev. Lett. 77, 960-963 (1996), doi:10.1103/PhysRevLett.77.960

7. Kloeden, P.E., Platen, E.: Numerical Solution of Stochastc Differential Equations. Springer, Berlin (1992)

8. Kramer, M.A., Kirsch, H.E., Szeri, A.J.: Pathalogical pattern formation and epileptic seizures. J. Roy. Soc. Interface 2, 113 (2005), doi:10.1098/rsif.2004.0028

Page: 18 job:Ch10_Wilson macro:svmult.cls date/time:8-Jul-2009/0:30 
9. Liley, D.T.J., Cadusch, P.J., Wright, J.J.: A continuum theory of electro-cortical activity. Neurocomp. 26-27, 795-800 (1999), doi:10.1016/S0925-2312(98)00149-0

10. Massimini, M., Huber, R., Ferrarelli, F., Hill, S., Tononi, G.: The sleep slow oscillation as a traveling wave. J. Neurosci. 24, 6862-6870 (2004), doi:10.1523/jneurosci.1318-04.2004

11. Numminen, J., Makela, J.P., Hari, R.: Distributions and sources of magnetoencephalographic K-complexes. Electroencephal. Clin. Neurophysl. 99, 544-555 (1996), doi:10.1016/S00134694(96)95712-0

12. Nunez, P.L.: The brain wave function: A model for the EEG. Math. Biosci. 21, 279-297 (1974), doi:10.1016/0025-5564(74)90020-0

13. Osan, R., Ermentrout, B.: Two dimensional synaptically generated travelling waves in a thetaneuron neural network. Neurocomp. 38-40, 789-795 (2001)

14. Rennie, C.J., Wright, J.J., Robinson, P.A.: Mechanisms for cortical electrical activity and emergence of gamma rhythm. J. Theor. Biol. 205, 17-35 (2000), doi:10.1006/jtbi.2000.2040

15. Robinson, P.A., Rennie, C.J., Rowe, D.L., O'Connor, S.C., Wright, J.J., Gordon, E., Whitehouse, R.W.: Neurophysical modeling of brain dynamics. Neuropsychopharmacol. 28, S74S79 (2003), doi:10.1038/sj.npp.1300143

16. Robinson, P.A., Rennie, C.J., Wright, J.J.: Propagation and stability of waves of electrical activity in the cerebral cortex. Phys. Rev. E 56, 826-840 (1997), doi:10.1103/PhysRevE.56.826

17. Steriade, M., Timofeev, I., Grenier, F.: Natural waking and sleep states: A view from inside neocortical neurons. J. Neurophysiol. 85, 1969-1985 (2001)

18. Steyn-Ross, D.A., Steyn-Ross, M.L., Sleigh, J.W., Wilson, M.T., Gillies, I.P., Wright, J.J.: The sleep cycle modelled as a cortical phase transition. J. Biol. Phys. 31, 547-569 (2005), doi:10.1007/s10867-005-1285-2

19. Steyn-Ross, M.L., Steyn-Ross, D.A., Sleigh, J.W.: Modelling general anaesthesia as a firstorder phase transition in the cortex. Progress in Biophysics and Molecular Biology 85, 369385 (2004), doi:10.1016/j.pbiomolbio.2004.02.001

20. Wilson, M.T., Sleigh, J.W., Steyn-Ross, D.A., Steyn-Ross, M.L.: General anesthetic-induced seizures can be explained by a mean-field model of cortical dynamics. Anesthesiology 104, 588-593 (2006), doi:10.1097/00000542-200603000-00026

21. Wilson, M.T., Steyn-Ross, D.A., Sleigh, J.W., Steyn-Ross, M.L., Wilcocks, L.C., Gillies, I.P.: The K-complex and slow oscillation in terms of a mean-field cortical model. J. Comp. Neurosci. 21, 243-257 (2006), doi:10.1007/s10827-006-7948-6

22. Wilson, M.T., Steyn-Ross, M.L., Steyn-Ross, D.A., Sleigh, J.W.: Predictions and simulations of cortical dynamics during natural sleep using a continuum approach. Phys. Rev. E 72, 051910 1-14 (2005), doi:10.1103/PhysRevE.72.051910

23. Wright, J.J., Liley, D.T.J.: Dynamics of the brain at global and microscopic scales: Neural networks and the EEG. Behav. Brain Sci. 19, 285-316 (1996) 


\section{Index}

Acetylcholine, 4

Adenosine, 4

\section{Bifurcation}

Hopf, 5-7

saddle-node, 15

Breathing mode, 8-10

Diffusive coupling, 8

EEG (electroencephalogram), 1, 7, 13

Eigenvalue, 6

EPSP (excitatory post-synaptic potential), 3

\section{Firing rate} sigmoidal function, 4

Hopf bifurcation, 5-7

IPSP (inhibitory post-synaptic potential), 3, 6

K-complex, 14-17

Macrocolumn, 2

Mean-field model, 1-4

limitations of, 1 macrocolumn, 2

Neurotransmitter

AMPA, 3

GABA, 3

Phase transition, 4

Phase-space trajectory, 14

PSP (post-synaptic potential), 2

REM (rapid-eye-movement), 5

Saddle-node bifurcation, 15

Seizure

epilepsy, 17

Sleep

rapid-eye-movement (REM), 5

slow-wave (SWS), 5

Spatial diffusion, 8

Spiral wave, 17

Stationary states, 4-5

SWS (slow-wave sleep), 5

Synaptic flux, 15

White noise, 2 